\title{
Characterizing exoplanets atmospheres with space photometry at optical wavelengths
}

\author{
Vivien Parmentier ${ }^{1,2,4, a}$, Tristan Guillot ${ }^{1}$, Adam P. Showman ${ }^{3}$, Jonathan Fortney ${ }^{4}$, and Mark Marley ${ }^{5}$ \\ 1 Laboratoire Lagrange, UMR 7293, Université de Nice-Sophia Antipolis, CNRS, Observatoire de la \\ Côte d'Azur, 06304 Nice Cedex 04, France \\ 2 Department of Planetary Sciences, Lunar and Planetary Laboratory,University of Arizona \\ 3 Department of Astronomy and Astrophysics, University of California, Santa Cruz, CA 95064, USA \\ 4 Sagan fellow \\ 5 NASA Ames Research Center, MS-245-3, Mofett Field, CA 94035
}

\begin{abstract}
Space photometry such as performed by Kepler and CoRoT provides exoplanets radius and phase curves with an exquisite precision. The phase curve constrains the longitudinal variation of the albedo and shed light on the horizontal distribution of clouds. The planet radius constraints thermal evolution of the planet, potentially unveiling its atmospheric composition. We present how the atmospheric circulation can affect the cloud distribution of three different planets, HD209458b, Kepler-7b and HD189733b based on three-dimensional models and analytical calculations. Then we use an analytical atmospheric model coupled to a state-of-the-art interior evolution code to study the role of $\mathrm{TiO}$ in shaping the thermal evolution and final radius of the planet.
\end{abstract}

\section{Introduction}

Space photometry allows the detection and characterization of planets and also shed light into their atmospheric characteristics. As seen in Fig. 1, known planets cover a wide range of the equilibrium temperature-rotation period-gravity parameter space, three parameters that shape the atmospheric circulation on these planets. Here we investigate the role of the atmospheric circulation in shaping the atmospheric composition, cloud distribution and radius evolution of irradiated exoplanets.

\section{Spatial variation of clouds in hot Jupiters}

Hints of clouds have been detected in the transit spectra of numerous hot Jupiters with different equilibrium temperatures and gravities (e.g. [2]). The presence of clouds in these distant atmospheres preclude the quantification of the bulk abundance of chemical species and also affects the gaseous composition of the atmosphere. Given the strong irradiation from their parent star, hot Jupiters posses deep radiative atmospheres. Nonetheless, the large day/night contrast in the irradiation they receive drives a powerful circulation that mixes material horizontally and vertically. The presence of clouds and their spatial variation is therefore influenced by the atmospheric circulation.

\subsection{Variability at the limb}

We perform three-dimensional simulations of the hot Jupiters HD209458b for which a small water signature has been observed with transit spectroscopy [3]. The simulation includes passive tracers that

\footnotetext{
a e-mail: vivien.parmentier@oca.eu
}

This is an Open Access article distributed under the terms of the Creative Commons Attribution License 4.0, which permits unrestricted use, distribution, and reproduction in any medium, provided the original work is properly cited. 


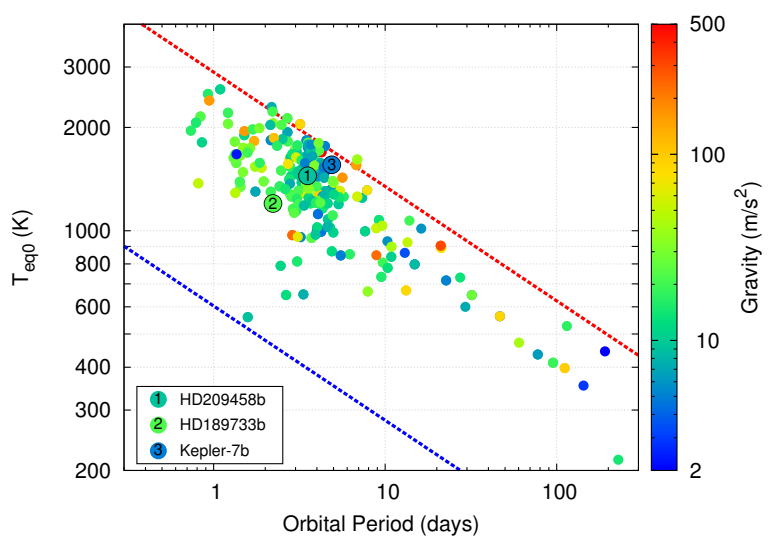

Fig. 1. Orbital period vs. equilibrium temperature for planets with a known mass and radius. Colors represent the gravity. Planets with orbital period less than $\approx$ 10days are believed to be tidally locked, with their rotation period equal to their orbital period (see [1])
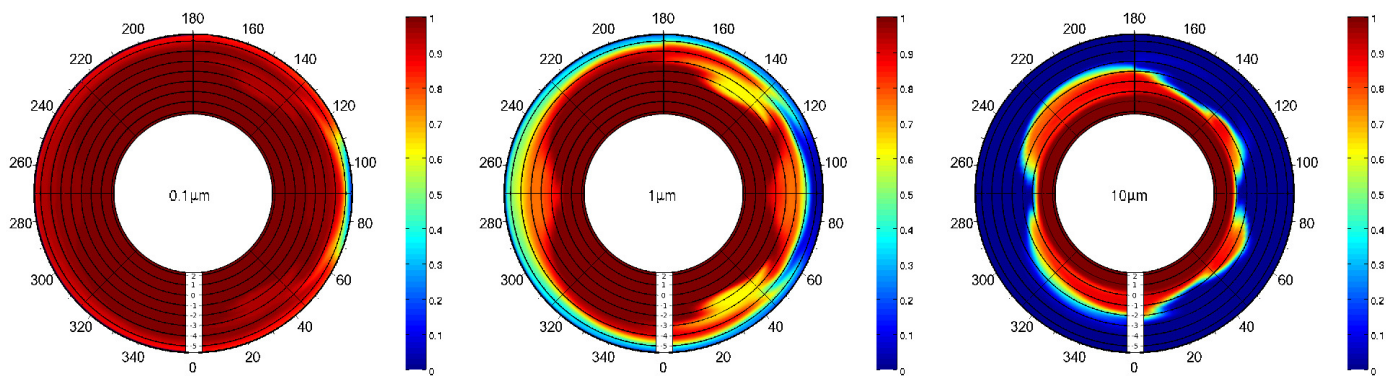

Fig. 2. Cloud abundance at the limb of HD209458b as predicted by a global circulation model. An abundance of 1 correspond to an abundance of the condensed gaseous species equal to it's deep abundance. The planet is shown as seen during transit, the black lines are isobars in logarithm of the pressure from $200 \mathrm{bar}$ to $1 \mu \mathrm{bar}$. The different panels assume different particle size for the clouds [4].

represent condensable species such as enstatite: the tracer field follows the fluid on the dayside and have a additional settling term on the nightside. The settling velocity is determined by the particle size, considered as a free parameter [4]. As shown in Fig. 2 the cloud abundance at the limb of the planet depends strongly on particle size: whereas large particles are confined below 10mbar, particles smaller than a micron are lifted up to the micro-bar layers, obscuring molecular signatures.

\subsection{Longitudinal variations}

Phase curves in the optical wavelength range can probe the longitudinal variation of the planet albedo. A shift of the maximum of the optical lightcurve after the secondary eclipse of numerous Kepler planets has been observed $[5,6]$ and interpreted as an increased cloud coverage west of the substellar point. Here use the analytical model developed in [7] to fit Kepler-7b's lightcurve. This model assumes an atmosphere homogeneous in latitude but with two zones of different single-scattering albedos in longitude: $\omega_{1}$ from longitude $-90^{\circ}$ to $\phi_{0}$ and $\omega_{2}$ from $\phi_{0}$ to $+90^{\circ}$. The scattering is assumed to be Lambertian. Our best fit is shown in Fig. 3 and corresponds to $\omega_{1}=1$, compatible with a cloudy atmosphere, $\omega_{2}=0.1$, compatible with a cloudless atmosphere and $\phi_{0}=-14^{\circ}$. Thus, clouds are expected to be located west of $-14^{\circ}$ of longitude. This matches the longitude where the condensation curve of the main condensable species (enstatite, forsterite and iron) cross the temperature profile obtained with a global circulation model [8]. 

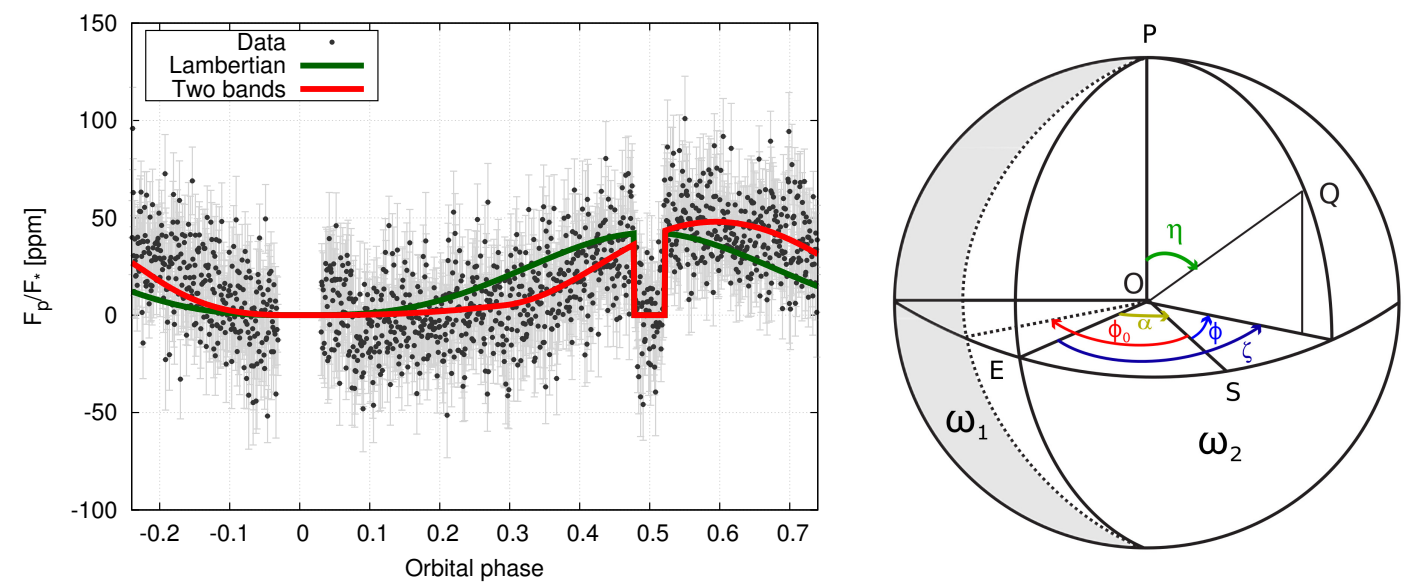

Fig. 3. The left panel show the Kepler light curve of Kepler-7b from [5] fitted with two different models assuming Lambert scattering: the first one for an homogeneous atmosphere, the second one with two bands of different albedos in longitude. The right panel describes the two-band model used in the left panel [7] (figure adapted from [9]).

\subsection{Latitudinal variations}

We performed three-dimensional simulations of HD189733b, the archetype of the cloudy planet [2], including passive tracers representing a condensable species that condenses everywhere above 1 bar in the planet (relevant for enstatite and forsterite for this planet). We find that the atmospheric circulation can produce large latitudinal gradients in the cloud abundance, leading to a banding with thick clouds at the poles and no clouds at the equator. Such a latitudinal cloud pattern should be observable with tens of eclipses of the brightest targets observed by PLATO.

\section{Atmospheric composition and radius evolution}

With space photometry, the radius of numerous planets can be determined with precision. For gas giant planets, the planet radius is a function of time: as the planet cools, it releases it's internal energy and shrinks. This process depends on the ability of the atmosphere to transport the internal flux from the planet interior toward outer space. The presence of radiatively active species in a planet atmosphere can change its energy balance and influence its evolution [10,11]. Particularly, in the hottest of the hot Jupiters, temperatures on the dayside are hot enough for titanium oxide to be present in gaseous phase, changing dramatically the heating and cooling rates of the atmosphere. However, TiO can condense and settle down on the nightside potentially leading to its depletion in the whole atmosphere. The efficiency of this cold trap depends on the strength of atmospheric circulation and the planet gravity among other parameters [12,4]. Planets in different regions of the parameter space described in Fig. 1 should have different abundance of $\mathrm{TiO}$ and thus experience a different evolution over time.

We use the analytical model of [13] to produce a grid of analytical models that matches closely the numerical predictions obtained for planets including $\mathrm{TiO} / \mathrm{VO}$ and planets where $\mathrm{TiO} / \mathrm{VO}$ has been removed from the atmosphere [14] (see Fig. 4). We use this model as a boundary condition to the internal structure model CEPAM [15] that calculates the radius evolution of a gaseous planet over time. As shown in Fig. 5, planets with $\mathrm{TiO}$ in their atmospheres are predicted to be a few percent smaller than planets without $\mathrm{TiO}$. This effect increases with increasing equilibrium temperature and with decreasing planet mass. Planets where the cold trapping of TiO is efficient (cold atmosphere, high gravity, weak circulation) should therefore have larger radius than planets where it is inefficient. As for now, no evidence for such difference has been found but has neither been ruled out, a situation that might change with the future yield of planets from CHEOPS, TESS and PLATO. 

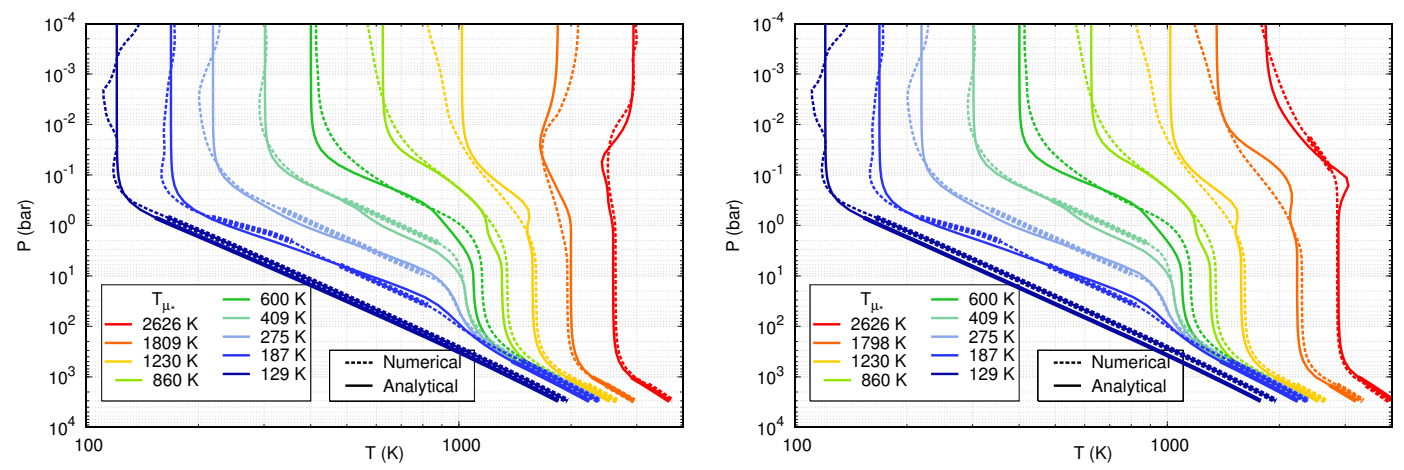

Fig. 4. Analytical (plain lines) and numerical (dotted lines) temperature profiles of irradiated planets atmosphere with (left panel) and without TiO (right panel).

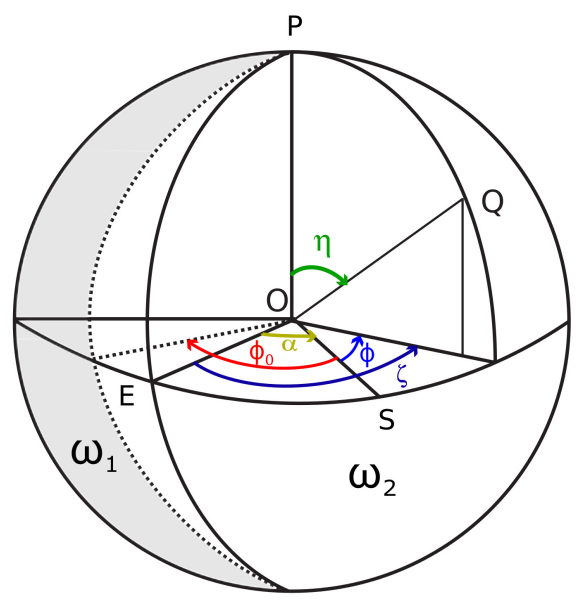

Fig. 5. Relative difference in radius expected between a 5 billion years old giant planet with $\mathrm{TiO}$ and without $\mathrm{TiO}$ in its atmosphere. Planets with $\mathrm{TiO}$ should be some percent smaller than planets without TiO.

\section{References}

1. V. Parmentier, A.P. Showman, J. de Wit, Experimental Astronomy (2014), 1401. 3673

2. F. Pont, D.K. Sing, N.P. Gibson, S. Aigrain, G. Henry, N. Husnoo, MNRAS (2013), 1210. 4163

3. D. Deming, A. Wilkins, P. McCullough, A. Burrows, J.J. Fortney, E. Agol, I. Dobbs-Dixon, N. Madhusudhan, N. Crouzet, J.M. Desert et al., ApJ 774, 95 (2013), 1302 . 1141

4. V. Parmentier, A.P. Showman, Y. Lian, A\&A 558, A91 (2013), 1301.4522

5. B.O. Demory, J. de Wit, N. Lewis, J. Fortney, A. Zsom, S. Seager, H. Knutson, K. Heng, N. Madhusudhan, M. Gillon et al., ApJ 776, L25 (2013), 1309. 7894

6. L.J. Esteves, E.J.W. De Mooij, R. Jayawardhana, ApJ 772, 51 (2013), 1305 . 3271

7. V. Parmentier, Ph.D. thesis (2014)

8. N. Lewis, V. Parmentier, A.P. Showman, J.J. Fortney, M.S. Marley, In prep. (2015)

9. N. Madhusudhan, A. Burrows, ApJ 747, 25 (2012), 1112 . 4476

10. J.J. Fortney, K. Lodders, M.S. Marley, R.S. Freedman, ApJ 678, 1419 (2008), 0710 . 2558

11. D.S. Spiegel, A. Burrows, ApJ 772, 76 (2013), 1303.0293

12. A.P. Showman, J.J. Fortney, Y. Lian, M.S. Marley, R.S. Freedman, H.A. Knutson, D. Charbonneau, ApJ 699, 564 (2009), 0809. 2089

13. V. Parmentier, T. Guillot, A\&A 562, A133 (2014), 1311.6597

14. V. Parmentier, T. Guillot, J.J. Fortney, M.S. Marley, ArXiv:1311.6322 (2014), 1311.6322

15. T. Guillot, P. Morel, A\&AS 109, 109 (1995) 